\title{
Analyticity of the Free Energy of a Closed 3-Manifold ${ }^{\star}$
}

\author{
Stavros GAROUFALIDIS ${ }^{\dagger}$, Thang T.Q. LE $\hat{E}^{\dagger}$ and Marcos MARIÑO ${ }^{\ddagger}$ \\ $\dagger$ School of Mathematics, Georgia Institute of Technology, Atlanta, GA 30332-0160, USA \\ E-mail:stavros@math.gatech.edu,letu@math.gatech.edu \\ ¥ Section de Mathématiques, Université de Genève, CH-1211 Genève 4, Switzerland \\ E-mail: Marcos.Marino@unige.ch
}

Received September 15, 2008, in final form November 06, 2008; Published online November 15, 2008

Original article is available at http://www.emis.de/journals/SIGMA/2008/080/

\begin{abstract}
The free energy of a closed 3-manifold is a 2-parameter formal power series which encodes the perturbative Chern-Simons invariant (also known as the LMO invariant) of a closed 3-manifold with gauge group $U(N)$ for arbitrary $N$. We prove that the free energy of an arbitrary closed 3-manifold is uniformly Gevrey-1. As a corollary, it follows that the genus $g$ part of the free energy is convergent in a neighborhood of zero, independent of the genus. Our results follow from an estimate of the LMO invariant, in a particular gauge, and from recent results of Bender-Gao-Richmond on the asymptotics of the number of rooted maps for arbitrary genus. We illustrate our results with an explicit formula for the free energy of a Lens space. In addition, using the Painlevé differential equation, we obtain an asymptotic expansion for the number of cubic graphs to all orders, stengthening the results of Bender-Gao-Richmond.
\end{abstract}

Key words: Chern-Simons theory; perturbation theory; gauge theory; free energy; planar limit; Gevrey series; LMO invariant; weight systems; ribbon graphs; cubic graphs; lens spaces; trilogarithm; polylogarithm; Painlevé I; WKB; asymptotic expansions; transseries; Riemann-Hilbert problem

2000 Mathematics Subject Classification: 57N10; 57M25

\section{Introduction}

\subsection{The free energy of a closed 3-manifold}

The free energy $F_{M}(\tau, \hbar)$ of a closed 3-manifold $M$ (defined in equation (1.7)) is a 2-parameter formal power series with rational coefficients in two variables $\tau$ and $\hbar^{2}$

$$
F_{M}(\tau, \hbar)=\sum_{g=0}^{\infty} F_{M, g}(\tau) \hbar^{2 g-2} \in \hbar^{-2} \mathbb{Q}\left[\left[\tau, \hbar^{2}\right]\right] .
$$

The variable $\hbar$ plays the role of Planck's constant, and the variable $\tau=N \hbar$ is the product of Planck's constant with $N$, the size of the gauge group $U(N)$. The free energy encodes the perturbative Chern-Simons invariant of $M$ along the trivial flat connection (also known as the LMO invariant of [41]) with gauge group $U(N)$ for arbitrary $N . F_{M, g}(\tau)\left(\operatorname{resp} . F_{M, 0}(\tau)\right)$ is often called the genus $g$-contribution (resp. planar limit) to the free energy. Perturbative Quantum Field Theory for a gauge group of fixed size $N$ typically leads to factorially divergent formal power series partly because there are factorially many Feynman diagrams, as was explained for example in [20]. It is a fundamental question how to give rigorous analytic meaning to these factorially divergent series and how to numerically evaluate them for example for QCD, and compare with well-tested experimental data. With this in mind, 't Hooft observed that when

\footnotetext{
${ }^{\star}$ This paper is a contribution to the Special Issue on Deformation Quantization. The full collection is available at http://www.emis.de/journals/SIGMA/Deformation_Quantization.html
} 
the size $N$ of the gauge is arbitrary, the Feynman diagrams of perturbative gauge theory organize themselves in ribbon graphs, i.e., abstract connected oriented surfaces of some genus $g$ with some nonzero number of boundary components; see [35]. Correspondingly, the free energy $F_{M}(\tau, \hbar)$ becomes a sum of power series $F_{M, g}(\tau)$ of a single variable that carries the contribution of the connected graphs of genus $g$. 't Hooft suggested that

Conjecture 1.1. There exists a disk $D_{M}$ that contains zero such that for all $g, F_{M, g}(\tau)$ is a power series analytic in $D_{M}$.

If the above conjecture holds, then $F_{M, g}(\tau)$ can be evaluated near $\tau=0$ by a convergent series. The above conjecture can be formulated for any gauge theory. 't Hooft's conjecture has been verified in some simple quantum-field theoretical models with $U(N)$ symmetry, such as matrix models (QFT in zero dimensions) and matrix quantum mechanics (QFT in one dimension); see $[12,10,16,17,55]$. Evidence for its validity in $N=4$ super Yang-Mills theory has also appeared recently; see $[9,8]$.

\section{$1.2 \quad$ A free energy of a knot}

In the world of Quantum Topology, the free energy of a 3-manifold is structurally similar to two other invariants of knots: namely the HOMFLY polynomial $H_{K}(t, q)$ of a knot $K$ and the Colored Jones function $J_{K}(q, n)$, colored by the $n$-dimensional irreducible representation of $\mathrm{SL}(2, \mathbb{C})$; see for instance [54]. The HOMFLY polynomial gives rise to an element of $\hbar^{-2} \mathbb{Q}\left[\left[\tau, \hbar^{2}\right]\right]$ using the substitution

$$
t=e^{\tau}=e^{N \hbar}, \quad q=e^{\hbar} .
$$

The same substitution works for the colored Jones function of a knot and is the content of the so-called loop expansion of the colored Jones function

$$
J_{K}(q, n)=\sum_{n=0}^{\infty} F_{K, n}(\tau) \hbar^{n} \in \mathbb{Q}[[\tau, \hbar]] .
$$

The above loop expansion is really perturbation theory of Chern-Simons theory along an Abelian flat connection on the knot complement, see [51]. For a detailed discussion on the meaning of the above expansion, see [51, 28, 22, 21]. Moreover, its planar limit $F_{K, 0}(\tau)$ can be identified with the inverse Alexander polynomial $\Delta_{K}$

$$
F_{K, 0}(\tau)=\frac{1}{\Delta_{K}\left(e^{\tau}\right)} .
$$

This is the content of the Melvin-Morton-Rozansky Conjecture, shown in [3]. In addition, for every $n \in \mathbb{N}$, we have

$$
F_{K, n}(\tau)=\frac{P_{K, n}\left(e^{\tau}\right)}{\Delta_{K}\left(e^{\tau}\right)^{2 n+1}},
$$

where $P_{K, n}(t) \in \mathbb{Z}\left[t^{ \pm 1}\right][51]$. Since $\Delta_{K}(1)=1$, it follows that the radius of convergence of $F_{K, n}(\tau)$ at $\tau=0$ is positive and independent of $n$.

\subsection{Power series uniformly Gevrey-1}

In order to state our results, we need to formalize the analytic properties of the free energy of a 3-manifold. Recall that a formal power series

$$
f(\hbar)=\sum_{n=0}^{\infty} a_{n} \hbar^{n}
$$


is Gevrey-s if there exists a positive constant $C>0$ such that

$$
\left|a_{n}\right| \leq C^{n} n !^{s}
$$

for all $n>0$.

Definition 1.1. Consider a formal power series

$$
f(x, \epsilon)=\sum_{n=-1}^{\infty} S_{n}(x) \epsilon^{n} \in \epsilon^{-1} \mathbb{C}[[x, \epsilon]]
$$

of two variables $(x, \epsilon)$. We say that $f(x, \epsilon)$ is Gevrey-1 with respect to $\epsilon$, uniformly with respect to $x=0$ (in short, $(x, \epsilon)$ Gevrey-1) if there exists a constant $C>0$ so that

$$
\left|\left[x^{k}\right] S_{n}(x)\right| \leq C^{n+k} n !
$$

for all $n, k \in \mathbb{N}$. Here, $\left[x^{k}\right] g(x)$ denotes the coefficient of $x^{k}$ in a power series $g(x)$.

Examples of power series $f(x, \epsilon)(x, \epsilon)$ Gevrey-1 are the WKB solutions of difference or differential equations with a small parameter; see for example [1], where the authors call such series uniformly pre-Borel summable. The loop expansion of a knot is $(\tau, \hbar)$ Gevrey-1; see [22].

Observe that if a power series $f(x, \epsilon)$ given in $(1.5)$ is $(x, \epsilon)$ Gevrey-1, then for all $n \in \mathbb{N}$, the formal power series $S_{n}(x)$ is analytic in a common neighborhood of $x=0$, independent of $n$.

\subsection{Statement of our results}

For a closed 3-manifold $M$, let $Z_{M}$ denote the LMO invariant of $M$, [41], which is an infinite power series of vertex-oriented trivalent graphs, and represents perturbation theory along the trivial flat connection of a 3-manifold. Given a metrized Lie algebra $\mathfrak{g}$, one can replace graphs by rational numbers, keeping track of their number of vertices, and thus create a power series $\left(W_{\mathfrak{g}} \circ Z_{M}\right)(\hbar) \in \mathbb{Q}[[\hbar]]$. Let $Z_{M}(N, \hbar)$ denote the LMO invariant of $M$, composed with the $\mathfrak{g l}_{N}$ weight system; see [41]. It is easy to see that $Z_{M}(N, \hbar)$ is a formal power series in $N$ and $\hbar$ with constant term 1 , and its logarithm

$$
F_{M}(\tau, \hbar)=\log Z_{M}(N, \hbar)
$$

which is by definition the free energy of $M$, can be written in the form (1.1), where $\tau=N \hbar$.

Theorem 1.1. For every closed 3 -manifold $M$, the free energy $F_{M}(\tau, \hbar)$ is $\left(\tau, \hbar^{2}\right)$ Gevrey-1.

Theorem 1.1 follows from combining a presentation for the LMO invariant given in Theorem 1.2 with the definition of the $\mathfrak{g l}_{N}$ weight system, together with a crucial estimate on the number of rooted maps in arbitrary genus obtained by Bender-Gao-Richmond following work of Goulden-Jackson; see [7, 32] and Corollary 4.1 below.

Theorem 1.2. For every closed 3-manifold $M$, we can write its $L M O$ invariant $Z_{M}$ in the form

$$
Z_{M}=\sum_{\Gamma} c_{\Gamma} \cdot \Gamma,
$$

where we are summing over the set of trivalent graphs $\Gamma$ and

$$
\left|c_{\Gamma}\right| \leq C_{M}^{n}
$$

for all $\Gamma$ of degree $n$, where $C_{M}>0$ is a constant that depends on $M$. 
Theorem 1.1 has the following corollary proving 't Hooft's conjecture.

Corollary 1.1. For every closed 3-manifold $M$, the power series $F_{M, g}(\tau)$ are analytic in a common neighborhood of $\tau=0$, independent of $g$.

We now explain what happens when we specialize $N$ to be a fixed natural number, e.g. $N=2$. In [20], the first two authors proved the following theorem for a fixed metrized Lie algebra $\mathfrak{g}$. Let $Z_{M}$ denote the LMO invariant of $M$ and let $W_{\mathfrak{g}}$ denote the corresponding weight system. Then, $\left(W_{\mathfrak{g}} \circ Z_{M}\right)(\hbar) \in \mathbb{Q}[[\hbar]]$.

Theorem 1.3 ([20], Theorem 3). For every metrized Lie algebra and every rational homology sphere $M,\left(W_{\mathfrak{g}} \circ Z_{M}\right)(\hbar)$ is Gevrey-1.

Corollary 1.2. Theorem 1.1 implies Theorem 1.3 for $\mathfrak{g}=\mathfrak{g l}_{N}$ for every fixed $N$.

\subsection{Some calculations}

As a concrete illustration of our results, we can compute the free energy of a Lens space. Let $L(d, b)$ denote the Lens space obtained by $d / b \in \mathbb{Q}$ surgery on the unknot in $S^{3}$.

Recall the $\alpha$-polylogarithm function

$$
\mathrm{Li}_{\alpha}(x)=\sum_{n=1}^{\infty} \frac{x^{n}}{n^{\alpha}}
$$

for $\alpha \in \mathbb{R}$, defined by the absolutely convergent series for $|x|<1$ and analytically continued in $\mathbb{C} \backslash\{0,1\}$. For a detailed discussion, see [48] and also [14]. Let $B_{n}$ denote the $n$th Bernoulli number defined by the generating series

$$
\frac{x}{e^{x}-1}=\sum_{n=0}^{\infty} \frac{B_{n}}{n !} x^{n} .
$$

Theorem 1.4. Consider a Lens space $M=L(d, b)$. Its free energy is given by

$$
F_{M, g}(\tau)=(2 g-1) \frac{B_{2 g}}{(2 g) !}\left(d^{2-2 g} \operatorname{Li}_{3-2 g}\left(e^{\tau / d}\right)-\operatorname{Li}_{3-2 g}\left(e^{\tau}\right)\right)+a_{g}(\tau)
$$

where

$$
a_{g}(\tau)= \begin{cases}-\frac{\tau^{2}}{2} \log d-\left(d^{2}-1\right) \zeta(3)+\lambda_{L(d, b)} \frac{\tau^{3}}{2} & \text { if } g=0, \\ \frac{\tau}{24}\left(1-d^{-1}\right)+\frac{1}{12} \log d-\lambda_{L(d, b)} \frac{\tau}{2} & \text { if } g=1, \\ 0 & \text { if } g \geq 2 .\end{cases}
$$

It follows that for all $g \geq 2$ (resp. $g=0,1), F_{M, g}$ has analytic continuation as a meromorphic (resp. multivalued analytic) function on $\mathbb{C} \backslash \frac{1}{d \mathbb{Z}^{*}(1)}\left(\right.$ resp. $\left.\mathbb{C} \backslash \frac{1}{d \mathbb{Z}(1)}\right)$, where

$$
\mathbb{Z}(1)=2 \pi i \mathbb{Z}, \quad \mathbb{Z}^{*}(1)=\mathbb{Z}(1) \backslash\{0\}
$$




\section{The LMO invariant and its $\ell^{\infty}$ Gromov norm}

\subsection{The $\ell^{\infty}$ Gromov norm}

The purpose of this section is to prove Theorem 1.2 extending the Gromov norm techniques from [20]. We will assume some familiarity with [20]. To begin with, the LMO invariant $Z_{M}$ of a closed 3-manifold takes value in a completed graded vector space $\mathcal{A}(\emptyset)$ of vertex-oriented trivalent graphs, modulo some linear homogeneous AS and IHX relations, where the degree of a graph is half the number of vertices. The LMO invariant gives a meaningful definition to the Chern-Simons perturbation theory along a trivial flat connection, and the trivalent graphs mentioned above as the Feynmann diagrams of a $\phi^{3}$ gauge theory with Chern-Simons action. For a detailed discussion, see [39]. The linear AS and IHX relations are the diagrammatic version of the antisymmetry and the Jacobi identity of the Lie algebra of the gauge theory.

Let $\mathcal{A}_{n}(\emptyset)$ denote the subspace of $\mathcal{A}(\emptyset)$ of degree $n$. Then, $\mathcal{A}_{n}(\emptyset)$ is a finite dimensional vector space spanned by the finite set of vertex-oriented trivalent graphs with $2 n$ vertices. Of course, this spanning set is not a basis, due to the linear AS and IHX relations. This motivates the following concept of the $\ell^{p}$ Gromov norm, extending the one in [20, Definition 1.1].

Definition 2.1. Consider a vector space $V$ and a spanning set $b$. Fix $p \in[1, \infty]$. For $v \in V$, define the $\ell^{p}$ norm by:

$$
|v|_{p}= \begin{cases}\inf \left(\sum_{j}\left|c_{j}\right|^{p}\right)^{1 / p} & \text { if } p \in[1, \infty), \\ \inf \max _{j}\left|c_{j}\right| & \text { if } p=\infty,\end{cases}
$$

where the infimum is taken over all presentations of the form $v=\sum_{j} c_{j} v_{j}, v_{j} \in b$.

We now apply the above definition to each of $\mathcal{A}_{n}(\emptyset)$ and combine them into a single power series.

\section{Definition 2.2.}

(a) Fix $p \in[1, \infty]$ and $n \in \mathbb{N}$ and consider the vector space $\mathcal{A}_{n}(\emptyset)$ spanned by the set $b$ of oriented trivalent graphs of degree $n$. For $v \in \mathcal{A}_{n}(\emptyset)$, we denote by $|v|_{p}$ the norm of $v$.

(b) If $v \in \mathcal{A}(\emptyset)$, we define

$$
|v|_{p}(\hbar)=\sum_{n=0}^{\infty}\left|\pi_{n}(v)\right|_{p} \hbar^{n} \in \mathbb{Q}[[\hbar]]
$$

where $\pi_{n}: \mathcal{A}(\emptyset) \longrightarrow \mathcal{A}_{n}(\emptyset)$ denotes the projection in $\mathcal{A}_{n}(\emptyset)$.

(c) We say that $v \in \mathcal{A}(\emptyset)$ has Gevrey-s $p$-norm if $|v|_{p}(\hbar)$ is Gevrey-s.

\section{$2.2 \quad$ A brief review of [20]}

The main result of [20] was the following theorem.

Theorem 2.1 ([20], Theorem 2). For every integral homology sphere $M$, the power series $\left|Z_{M}\right|_{1}(\hbar) \in \mathbb{Q}[[\hbar]]$ is Gevrey-1.

Theorem 2.1 is a convergence property of a universal finite type invariant of homology spheres, and uses a well-known universal finite type invariant of links in $S^{3}$, namely the Kontsevich integral. For an introduction to the notion of finite type invariants of knots and 3-manifolds, see $[2,50]$. Since our proof of Theorem 1.2 will use some of the ideas of the proof of Theorem 2.1, we begin by recalling the proof of Theorem 2.1, which goes as follows. First, we begin with the following description of the LMO invariant from [5]. 
- Fix a presentation of an integral homology sphere $M$ as surgery on a unit-framed boundary link $L$ in $S^{3}$.

- Choose a presentation of $L$ as the closure of a framed string link $T$.

- Consider the normalized Kontsevich integral $\check{Z}_{T}$, which takes values in the completed $\mathbb{Q}$ vector space $\mathcal{A}\left(\star_{X}\right)$ of vertex-oriented Jacobi diagrams with legs colored by a set $X$ in 1-1 correspondence with the components of $T$.

- Perform formal diagrammatic Gaussian integration

$$
\int d X: \mathcal{A}\left(\star_{X}\right) \longrightarrow \mathcal{A}(\emptyset)
$$

to $\check{Z}_{T}$. I.e., separate out the strut part of $\check{Z}_{T}$ (which is an exponential of Jacobi diagrams with no trivalent vertices) and join the legs of the remaining diagrams using the inverse linking matrix of the strut part.

- Finally, define

$$
Z_{M}=\frac{\int \check{Z}_{L} d X}{\left(\int \check{Z}_{U^{+}} d X\right)^{\sigma_{+}(L)}\left(\int \check{Z}_{U^{-}} d X\right)^{\sigma_{-}(L)}},
$$

where $U^{ \pm}$denotes the \pm 1 framed unknot in $S^{3}$ and $\sigma_{ \pm}(L)$ denotes the number of positive (resp. negative) eigenvalues of the linking matrix of $L$.

Next, we extend our notion of $\ell^{p}$ norm to all intermediate spaces of Jacobi diagrams (with or without skeleton, and with or without symmetrized legs).

Next, we show that the Kontsevich integral is Gevrey-0.

Theorem 2.2 ([20], Theorem 8). For every framed tangle $T,\left|Z_{T}\right|_{1}(\hbar) \in \mathbb{R}[[\hbar]]$ is Gevrey-0.

This follows from the definition of the Kontsevich integral using the $\mathrm{KZ}$ associator, together with the following lemma.

Lemma 2.1 ([20], Proposition 2.6). If $U$ denotes the zero-framed unknot, then $\left|Z_{U}\right|_{1}(\hbar) \in$ $\mathbb{R}[[\hbar]]$ is Gevrey- 0 .

Finally we use the following key lemma.

Lemma 2.2 ([20], Lemma 2.10). For every boundary string link $T$ with framing \pm 1 , $\left|\int \check{Z}_{T} d X\right|_{1}(\hbar) \in \mathbb{R}[[\hbar]]$ is Gevrey-1.

The condition on the string link being a boundary one is to ensure that the strutless part of $\check{Z}_{T}$ contains no trees; see [33]. Lemma 2.2 takes care of the normalization of the LMO invariant given by (2.3). Ignoring technicalities, the main point in the proof of Lemma 2.2 is the following.

If $G$ is uni-trivalent graph of degree $n+k$ (i.e., with $2 n+2 k$ vertices) with $2 k$ legs, then there are $(2 k-1) ! !=1 \cdot 3 \cdot 5 \cdots(2 k-1)$ ways to pair the legs of $G$ together. If $G$ has no tree components, then $k \leq n$ thus

$$
(2 k-1) ! ! \leq(2 n-1) ! ! \leq C^{n} n ! .
$$

Remark 2.1. If we replace $|\cdot|_{1}$ by $|\cdot|_{p}$ for $p \in[1, \infty)$, Theorems $2.1,2.2$ and Lemmas 2.1, 2.2 remain true.

In the next section we discuss what happens when we use the $|\cdot|_{\infty}$ rather than the $|\cdot|_{1}$ norm. 


\subsection{Proof of Theorem 1.2}

We now show how to modify the statements of the previous section in order to show the following reformulation of Theorem 1.2. In that theorem we need to fix a closed 3-manifold. When $M$ has positive Betti number, the LMO invariant simplifies a lot, and it is possible to give a direct proof by an explicit formula. For example, when the first Betti number $b_{1}(M)$ is greater than 3 , then the LMO invariant vanishes and Theorem 1.2 obvious holds. When $1<b_{1}(M) \leq 3$, the LMO invariant can be directly computed, see [34, 50]. When $b_{1}(M)=1$, the LMO invariant is computed by [24] in terms of the Alexander polynomial of $M$, and Theorem 1.2 follows immediately. Thus, it suffices to assume that $M$ is a rational homology sphere, and further (using the multiplicative behavior of the LMO with respect to connected sums), it suffices to assume that $M$ is obtained by surgery on a rationally framed boundary link in $S^{3}$. In that case, the LMO invariant can be computed via the Aarhus integral.

Theorem 2.3. For every rational homology sphere $M,\left|Z_{M}\right|_{\infty}(\hbar) \in \mathbb{R}[[\hbar]]$ is Gevrey-0.

This theorem follows from the following.

Theorem 2.4. For every framed tangle $T,\left|Z_{T}\right|_{\infty}(\hbar) \in \mathbb{R}[[\hbar]]$ is Gevrey-0.

This follows easily from Theorem 2.2 and the fact that $|v|_{\infty} \leq|v|_{1}$ for all $v \in \mathcal{A}_{n}(\emptyset)$.

Lemma 2.3. For every framed string link $T$ with invertible linking matrix $\left|\int \check{Z}_{T} d X\right|_{\infty}(\hbar) \in$ $\mathbb{R}[[\hbar]]$ is Gevrey-0.

The proof of this lemma is as follows. First we write $\check{Z}_{T}$ as follows, using Theorem 2.4

$$
\check{Z}_{T}=\exp \left(\left.\frac{1}{2} \sum_{i j} l_{i j}\right|_{i} ^{j}\right) \sum_{\Gamma} c_{\Gamma} \cdot \Gamma,
$$

where $\left(l_{i j}\right)$ is the linking matrix of $T,\left.\right|_{i} ^{j}$ is a strut colored by the components $i$ and $j$ of $T$, the summation is over the set of uni-trivalent graphs with no strut components and legs colored by the components of $T$ and

$$
\left|c_{\Gamma}\right| \leq C^{\operatorname{deg}(\Gamma)}
$$

for some constant $C>0$. If $G$ is a trivalent graph of degree $n$, it has $3 n$ edges. If $G$ comes from pairing the legs of a uni-trivalent graph $\Gamma$, then $\Gamma$ is obtained from $G$ by cutting some (let's say $k \leq 3 n$ ) of the edges of $G$ in half. Such a uni-trivalent graph $\Gamma$ has degree $n+k$ (i.e., $2 n+2 k$ vertices) and $2 k$ legs and its coefficient in $\check{Z}_{T}$ is bounded by $C^{n+k} \leq C^{4 n}$. Since $G$ has $3 n$ edges, there are at most $2^{3 n}$ such graphs $\Gamma$. Thus, pairing the legs of the strutless part of $\check{Z}_{T}$, we obtain that

$$
\int \check{Z}_{T} d X=\sum_{G} c_{G} \cdot G
$$

where

$$
\left|c_{G}\right| \leq C^{\prime \operatorname{deg}(G)}
$$

for some $C^{\prime}>0$. This concludes the proof of Theorem 1.2. 


\section{$3 \quad$ The $\mathfrak{g l}_{N}$ weight system}

The LMO invariant of a closed 3-manifold takes values in a completed vector space $\mathcal{A}(\emptyset)$ spanned by trivalent graphs. To get a numerical invariant with values in $\mathbb{Q}[[\hbar]]$, we need to replace every graph by a combinatorial weight. This is exactly, what a weight system does. More precisely, given a Lie algebra $\mathfrak{g}$ with an invariant inner product, there is a weight system $\mathbb{Q}$-linear map:

$$
W_{\mathfrak{g}}: \mathcal{A}(\emptyset) \longrightarrow \mathbb{Q}[[\hbar]]
$$

discussed at great length in [2]. In [2], the following description of $W_{\mathfrak{g}}$ is given.

$$
W_{\mathfrak{g l}_{N}}: \mathcal{A}(\emptyset) \longrightarrow \mathbb{Q}[[N, \hbar]]
$$

is defined by:

$$
D \longrightarrow \sum_{M}(-1)^{s_{M}} N^{b_{D, M}} \hbar^{2 g_{D, M}-2+b_{D, M}}
$$

where

- the sum is over all possible markings $M$ of the trivalent vertices of $D$ by 0 or 1 ,

- $s_{M}$ is the sum, over the set of trivalent vertices, of the values of $M$,

- $\Sigma_{D, M}$ denotes the $X$-marked surface obtained by thickening the trivalent vertices of $D$ as follows:
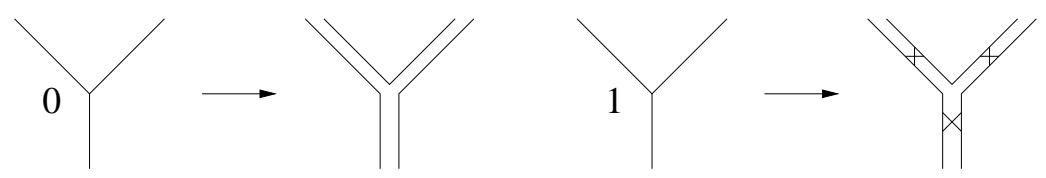

and thickening the edges of $D$, and connecting up into to a surface. It turns out that $\Sigma_{D, M}$ is well-defined, oriented and perhaps disconnected,

- $g_{D, M}$ and $b_{D, M}$ denote the genus (i.e., sum of the genera of its connected components) and the number of boundary components of $\Sigma(D, M)$,

- it turns out that the degree of $D$ is $2 g_{D, M}-2+b_{D, M}$.

For example, we have

$$
W_{\mathfrak{g l}_{N}}(\Theta)=2\left(N^{3}-N\right) \hbar
$$

where $\Theta$ is the obvious planar trivalent graph with counterclockwise cyclic order at each vertex. The next lemma follows directly from the above definition of the $W_{\mathfrak{g l}_{N}}$ weight system.

Lemma 3.1. If $\Gamma$ is a connected trivalent graph of degree $n$, then

$$
W_{\mathfrak{g l}_{N}}(\Gamma)=p_{\Gamma}(N) \hbar^{n}
$$

where $p_{\Gamma}(N) \in \mathbb{Z}[N]$ is a polynomial in $N$ of degree at most $n+2$ and $\ell^{1}$-norm at most $2^{n}$. 


\section{Asymptotics of the number of rooted maps of arbitrary genus}

In a series of papers in various collaborations, Bender et al studied the exact and asymptotic number of rooted maps on a surface; see [6,7]. Recall that a map $(G, S)$ is a graph $G$ embedded in a connected, oriented, closed topological surface $S$ in such a way that each maximal connected component of $S \backslash G$ is a topological disk. A map is rooted if an edge, a direction along the edge, and a side of the edge are distinguished. Let $T_{g}(n)$ denote the number of $n$-edged rooted maps on a surface of genus $g$. In [6], Bender-Canfield, using generating functions, and the Darboux-Polya enumeration method, give the following asymptotic expansion of $T_{g}(n)$.

Theorem 4.1 ([6]). For $g$ fixed and $n \longrightarrow \infty$ we have

$$
T_{g}(n) \sim t_{g} n^{5(g-1) / 2} 12^{n},
$$

where the constants $t_{g}$ are computable via computable non-linear recursions. In particular,

$$
t_{0}=\frac{2}{\sqrt{\pi}}, \quad t_{1}=\frac{1}{24}, \quad t_{2}=\frac{7}{4320 \sqrt{\pi}} .
$$

Many interesting families of maps satisfy asymptotic formulas of the form

$$
\alpha t_{g}(\beta n)^{5(g-1) / 2} \gamma^{n} \text {. }
$$

This is discussed extensively for example in [55, Section 4]; see also [29, 30]. For over 20 years, the constants $t_{g}$ remained hard to compute or estimate, partly due to the complexity of their nonlinear recursion. A breakthrough was achieved recently. Using work of Goulden-Jackson [32] on the KP hierarchy, Bender-Gao-Richmond simplified the non-linear recursion relation for $t_{g}$, and obtained the following.

Theorem 4.2 ([7]). We have

$$
t_{g} \sim \frac{40 \sin (\pi / 5) K}{\sqrt{2 \pi}}\left(\frac{1440 g}{e}\right)^{-g / 2},
$$

where $K=0.10486898772254091800 \ldots$ is a constant.

The numerical value of $K$ was given incorrectly at [7]. An exact value of $K$ is given in equation (A.16) in Section A below. For our purposes, it suffices to note that a trivalent graph of degree $n$ has $3 n$ edges. Taking into account the choice of a root, it follows that

Corollary 4.1. There are at most $12 n T_{g}(3 n)$ connected trivalent graphs of genus $g$, where $T_{g}(n)$ satisfies (4.1) and (4.2).

Remark 4.1. Theorem 4.1 has an analogous statement (with a different constant $p_{g}$ instead of $t_{g}$ ) for rooted graphs in unoriented surfaces; see [6, Theorem 1]. Without doubt, there is an asymptotic expansion for $p_{g}$ analogous to $t_{g}$. In fact, one can write down explicitly a non-linear recursion relation that $p_{g}$ ought to satisfy, and study its exact asymptotic behavior. This will be discussed in a separate publication; see [27].

\section{Proofs}

\subsection{Proof of Theorem 1.1}

Let $Z_{M}(N, \hbar)$ denote the LMO invariant of $M$, composed with the weight system of $\mathfrak{g l}_{N}$. Then, we can write

$$
\log Z_{M}(N, \hbar)=\sum_{2 g-2+d>0, d>0} a_{M, g, d} N^{d} \hbar^{2 g-2+d} .
$$


Recall that the free energy of $M$ is defined by (1.7)

$$
F_{M}(\tau, \hbar)=\log Z_{M}(N, \hbar),
$$

where $\tau=N \hbar$. It follows that the free energy has the form (1.1) where

$$
F_{M, g}(\tau)=\sum_{d: 2 g-2+d>0, d>0} a_{M, g, d} \tau^{d} \in \mathbb{Q}[[\tau]] .
$$

On the other hand, Theorem 1.2 implies that we can write

$$
\log Z_{M}=\sum_{\Gamma} c_{\Gamma} \cdot \Gamma
$$

where the sum is over a set of connected trivalent graphs, where there exists a constant $C$ such that

$$
\left|c_{\Gamma}\right| \leq C^{n}
$$

if $\Gamma$ has degree $n$. Applying the $W_{\mathfrak{g l}_{N}}$ weight system, and using Lemma 3.1 it follows that

$$
F_{M}(\tau, \hbar)=\sum_{n=1}^{\infty} \sum_{\Gamma} c_{\Gamma} \cdot p_{\Gamma}(N) \hbar^{n},
$$

where the $\Gamma$ summation is over a set of connected trivalent graphs of degree $n$. Thus,

$$
a_{M, g, d}=\sum_{\Gamma} c_{\Gamma} \cdot\left[N^{d}\right] p_{\Gamma}(N),
$$

where the sum is over the set of connected trivalent graphs $\Gamma$ of degree $n=2 g-2+d$ that embed on a surface of genus $g$. Lemma 3.1 estimates the coefficients $\left[N^{d}\right] p_{\Gamma}(N)$ and since $2 g-2+d=n$, it implies that

$$
\left|a_{M, g, d}\right| \leq C_{1}^{n} \sum_{\Gamma}\left|c_{\Gamma}\right|
$$

for some constant $C_{1}>0$. Equation (5.3) together with $2 g-2+d=n$ implies that

$$
\left|a_{M, g, d}\right| \leq C_{2}^{n} \sum_{\Gamma} 1
$$

for some constant $C_{2}>0$. The above sum is over the set of trivalent graphs of degree $n=$ $2 g-2+d$ (and thus, $3 n$ edges) that embed on a surface of genus $g$. Corollary 4.1 implies that

$$
\begin{aligned}
\sum_{\Gamma} 1 & \leq 8 n T_{g}(3 n) \leq C_{1}^{n}\left(\frac{(3 n)^{5}}{g}\right)^{g / 2} \leq C_{2}^{n} \frac{(2 g-2+d)^{5 g / 2}}{g^{g / 2}} \\
& =C_{2}^{n} \frac{(2 g-2+d)^{5 g / 2}}{(2 g)^{5 g / 2}} \frac{(2 g)^{5 g / 2}}{g^{g / 2}} .
\end{aligned}
$$

Since $(1+a / n)^{n} \leq e^{a}$, we have

$$
\frac{(2 g-2+d)^{5 g / 2}}{(2 g)^{5 g / 2}} \leq e^{5(d-2) / 4} .
$$

Moreover,

$$
\frac{(2 g)^{5 g / 2}}{g^{g / 2}} \leq C_{3}^{g}(2 g) !
$$

Since $n=2 g-2+d$, it follows that

$$
\left|a_{M, g, d}\right| \leq C_{3}^{g+d}(2 g) !
$$

and concludes the proof of Theorem 1.1. 


\subsection{Proof of Corollary 1.2}

Fix a natural number $N \in \mathbb{N}$. It suffices to prove that $\left(W_{\mathfrak{g l}_{N}} \circ \log Z_{M}\right)(\hbar) \in \mathbb{Q}[[\hbar]]$ is Gevrey-1. Equation (5.1) implies that

$$
\left[\hbar^{n}\right]\left(W_{\mathfrak{g l}_{N}} \circ \log Z_{M}\right)(\hbar)=\sum_{g, d: 2 g-2+d=n} a_{M, g, d} N^{d} .
$$

The proof of Theorem 1.1 implies that there exists $C>0$ so that

$$
\left|a_{M, g, d}\right| \leq C^{g+d}(2 g) !
$$

Since $2 g-2+d=n, C^{g+d} \leq C_{1}^{3 n / 2}=C_{2}^{n}$ and $(2 g) ! \leq C_{3}^{n} n !$. Thus,

$$
\left|\operatorname{coeff}\left(\hbar^{n},\left(W_{\mathfrak{g l}_{N}} \circ \log Z_{M}\right)(\hbar)\right)\right| \leq C_{4}^{n} n ! \sum_{g, d: 2 g-2+d=n} N^{d} \leq C_{4}^{n} n ! N^{n+2}(n+2) .
$$

Since $N$ is fixed, it follows that

$$
\left|\left[\hbar^{n}\right]\left(W_{\mathfrak{g l}_{N}} \circ \log Z_{M}\right)(\hbar)\right| \leq C^{n} n !
$$

which concludes the proof of Corollary 1.2.

\section{The free energy of a Lens space}

In this section we will prove Theorem 1.4. The next proposition computes the image of the LMO invariant of a Lens space $L(d, b)$ under the weight system $W_{\mathfrak{g}}$.

Let $\mathfrak{g}$ denote a metrized Lie algebra with inner product $\langle\cdot, \cdot\rangle$ and let $\Phi_{+}$denote the positive roots of $\mathfrak{g},\left|\Phi_{+}\right|$denote the cardinality of $\Phi_{+}$and $\rho$ denote half the sum of the positive roots of $\mathfrak{g}$. Let $c_{\mathfrak{g}}$ denote the product of the quadratic Casimir of $\mathfrak{g}$ with the dimension of $\mathfrak{g}$. Let $\lambda_{M}$ denote Casson invariant.

Proposition 6.1. With the above assumptions we have

$$
\left(W_{\mathfrak{g}} \circ Z_{L(d, b)}\right)(\hbar)=\exp \left(\frac{\lambda_{L(d, b)}}{4} c_{\mathfrak{g}} \hbar\right) d^{\left|\Phi_{+}\right|} \prod_{\alpha \in \Phi_{+}} \frac{\sinh ((\alpha, \rho) \hbar /(2 d))}{\sinh ((\alpha, \rho) \hbar / 2)} \in \mathbb{Q}[[\hbar]] .
$$

Proof. There are two proofs of this proposition. One proof uses

(a) The existence of the Ohtsuki series (which come from the Reshetikhin-Tuarev invariants of 3-manifolds). This was shown by Ohtsuki in [49] for PSU(2), and by the second author in [42] for $\operatorname{PSU}(N)$ and more generally in [43] for all simple Lie algebras.

(b) The computation of the Ohtsuki series for Lens spaces, done by Takata in [52].

(c) The identification of the left hand side of (6.1) with the Ohtsuki series. This was the result of the unpublished fourth part of [5] and independently an unpublished work of the second author that was recently completed by Kuriya [40], see also [50].

An alternative proof uses the computation of the LMO invariant for Lens spaces by Bar-Natan and Lawrence [4]. Let us give the details of this proof. We will use the notation from [4]. In [4, Proposition 5.1] it is shown that the LMO invariant of $L(d, b)$ is given by:

$$
Z_{L(d, b)}=\exp \left(\frac{-S(b / d)}{48} \Theta\right) \frac{\left\langle\Omega_{x}, \Omega_{x / d}\right\rangle_{x}}{\left\langle\Omega_{x}, \Omega_{x}\right\rangle_{x}} .
$$


Moreover, $-S(b / d)=12 \lambda_{L(b, d)}$. Now, we apply the weight system $W_{\mathfrak{g}}$. We have

$$
\begin{aligned}
& W_{\mathfrak{g}}(\Theta)(\hbar)=c_{\mathfrak{g}} \hbar, \\
& W_{\mathfrak{g}}\left(\left\langle\Omega_{x}, \Omega_{x}\right\rangle_{x}\right)(\hbar)=\prod_{\alpha \in \Phi_{+}} \frac{\sinh ((\alpha, \rho) \hbar / 2)}{(\alpha, \rho) \hbar / 2}
\end{aligned}
$$

and

$$
W_{\mathfrak{g}}\left(\left\langle\Omega_{x}, \Omega_{x / d}\right\rangle_{x}\right)(\hbar)=W_{\mathfrak{g}}\left(\left\langle\Omega_{x}, \Omega_{x}\right\rangle_{x}\right)(\hbar / d) .
$$

The result follows.

\subsection{Some special functions}

Let us introduce some auxiliary functions which have already appeared in the LMO invariant of the Lens spaces and which are important ingredients of the proof of Theorem 1.4. Consider the functions $f$ and $\widetilde{f}$ defined by

$$
\tilde{f}(x):=\frac{\sinh (x / 2)}{x / 2}, \quad \text { and } \quad f(x):=\log \tilde{f}(x) .
$$

It is known that

$$
f(x)=\sum_{k=1}^{\infty} b_{2 k} x^{2 k}=\frac{x^{2}}{24}-\frac{x^{4}}{2880}+\frac{x^{6}}{181440}+\cdots,
$$

where $b_{2 k}$ 's are the modified Bernoulli numbers and are related to the ordinary Bernoulli numbers $B_{k}$ as follows:

$$
b_{2 k}=\frac{B_{2 k}}{2 k(2 k) !} \text {. }
$$

The first polylogarithm is given by

$$
\operatorname{Li}_{1}(x)=-\log (1-x) .
$$

The relation between $f(x)$ and $\mathrm{Li}_{1}$ is the following

$$
f(x)=-\operatorname{Li}_{1}\left(e^{x}\right)-\frac{x}{2}-\log (-x) .
$$

The left hand side is an analytic function in the unit disk $|x|<1$ with an analytic continuation as a multivalued analytic function in $\mathbb{C} \backslash 2 \pi i(\mathbb{Z} \backslash\{0\})$. The right hand side is well-defined in the unit disk minus $[0,1)$, and extends as a continuous function over the cut $[0,1)$. Then, both sides agree on the unit disk $|x|<1$. Equation (6.3) follows from the following easy computation

$$
f(x)=\log \left(\frac{e^{x / 2}-e^{-x / 2}}{x}\right)=\log \left(\frac{e^{-x / 2}}{-x}\left(1-e^{x}\right)\right)
$$

and equation (6.2).

From the power series definition of the polylogarithm, it is easy to see that for all $\alpha \in \mathbb{R}$ we have

$$
\frac{d}{d x} \operatorname{Li}_{\alpha}\left(e^{x}\right)=\operatorname{Li}_{\alpha-1}\left(e^{x}\right) .
$$

Moreover, when $\alpha$ is a negative integer, then

$$
\operatorname{Li}_{\alpha}(x)=\frac{P_{\alpha}(x)}{(1-x)^{-\alpha+1}},
$$

where $P_{\alpha}(x) \in \mathbb{Z}[x]$ is a palindromic polynomial of degree $-\alpha$. It follows that for $\alpha$ a negative integer, the function $\operatorname{Li}_{\alpha}\left(e^{\tau}\right)$ is an even meromorphic function on $\mathbb{C}$ with poles at $\mathbb{Z}(1)$, where the latter set is defined in (1.13). 


\subsection{Proof of Theorem 1.4}

This section is devoted to the proof of Theorem 1.4. Let us observe that the $\mathfrak{g l}_{N}$ and the $\mathfrak{s l}_{N}$ weight system agree on all nonempty trivalent graphs (since the structure constants of an Abelian Lie algebra vanish, and are placed at the trivalent vertices of a trivalent graph), and that the logarithm of the LMO invariant of a closed 3-manifold is a series of nonempty connected trivalent graphs. Therefore, to compute the free energy, we can work with the $\mathfrak{s l}_{N}$ weight system. Recall that the set $\Phi_{+}$of positive roots of $\mathfrak{s l}_{N}$ has $N(N-1) / 2$ elements. From Proposition 6.1 one has

$$
\left(W_{\mathfrak{s l}_{N}} \circ Z_{L(d, b)}\right)(\hbar)=e^{\frac{\lambda L(d, b)}{12} c_{\mathfrak{s} \mathfrak{l}_{N}} \hbar} \prod_{\alpha \in \Phi_{+}} \frac{\tilde{f}((\alpha, \rho) \hbar / d)}{\tilde{f}((\alpha, \rho) \hbar)} .
$$

Taking the logarithm, we get

$$
F_{M}(\tau, \hbar)=\hbar \frac{\lambda_{L(d, b)}}{12} c_{\mathfrak{s l}}+\sum_{\alpha \in \Phi_{+}}(f((\alpha, \rho) \hbar / d)-f((\alpha, \rho) \hbar)) .
$$

Note that

$$
\hbar \frac{\lambda_{L(d, b)}}{4} c_{\mathfrak{s l}_{N}}=\frac{\lambda_{L(d, b)}}{2} \hbar\left(N^{3}-N\right)=\frac{\lambda_{L(d, b)}}{2}\left(\frac{\tau^{3}}{\hbar^{2}}-\tau\right) .
$$

When $\alpha$ runs through the set $\Phi_{+},(\alpha, \rho)$ takes the value $j$ the total of $N-j$ times, for every $j=1, \ldots, N-1$. Hence

$$
F_{M}(\tau, \hbar)=\hbar \frac{\lambda_{L(d, b)}}{12} c_{\mathfrak{g}}+\sum_{j=1}^{N-1}((N-j) f(j \hbar / d)-(N-j) f(j \hbar)) .
$$

Now we have

$$
\sum_{j=1}^{N-1}(N-j) f(j \hbar)=\sum_{j=1}^{N-1}(N-j) \sum_{k=1}^{\infty} b_{2 k} j^{2 k} \hbar^{2 k}=\sum_{k=1}^{\infty} b_{2 k} \hbar^{2 k} \sum_{j=1}^{N-1}(N-j) j^{2 k}
$$

Using the well-known identity expressing the sum of powers by a Bernoulli polynomial

$$
\sum_{j=1}^{N-1} j^{k}=\frac{1}{k+1} \sum_{s=0}^{k}\left(\begin{array}{c}
k+1 \\
s
\end{array}\right) B_{s} N^{k+1-s}
$$

it follows that

$$
\sum_{j=1}^{N-1}(N-j) j^{2 k}=\sum_{g=0}^{k} \frac{(2 k) !(1-2 g)}{(2 g) !(2 k+2-2 g) !} B_{2 g} N^{2 k+2-2 g} .
$$

Therefore, using $N=\tau / \hbar$, we get

$$
\begin{aligned}
\sum_{j=1}^{N-1}(N-j) f(j \hbar) & =\sum_{k=1}^{\infty} b_{2 k} \hbar^{2 k}\left\{\sum_{g=0}^{k} \frac{(2 k) !(1-2 g)}{(2 g) !(2 k+2-2 g) !} B_{2 g} N^{2 k+2-2 g}\right\} \\
& =\sum_{g=0}^{\infty} \frac{(1-2 g) B_{2 g} \hbar^{2-2 g}}{(2 g) !} \sum_{k=\max \{g, 1\}}^{\infty} \frac{(2 k) !}{(2 k+2-2 g) !} b_{2 k} \tau^{2 k+2-2 g}
\end{aligned}
$$




$$
=\sum_{g=0}^{\infty} \frac{(1-2 g) B_{2 g} \hbar^{2-2 g}}{(2 g) !} \sum_{l=0}^{\infty} \frac{(2 l+2 g) !}{(2 l+2) !} b_{2 g+2 l} \tau^{2 l+2}
$$

with a minor variation when $g=0$. Let us define the auxiliary functions $F_{g}$ by

$$
F_{g}(\tau):=\sum_{l=0}^{\infty} \frac{(2 l+2 g) !}{(2 l+2) !} b_{2 g+2 l} \tau^{2 l+2} .
$$

Then, equations $(6.6),(6.7)$ and $(6.8)$ and the replacement of $(\hbar, \tau)$ by $(\hbar / d, \tau / d)$ imply that

$$
F_{M, g}(\tau)=(1-2 g) \frac{B_{2 g}}{(2 g) !}\left(d^{2-2 g} F_{g}(\tau / d)-F_{g}(\tau)\right)+\frac{\lambda_{L(d, b)}}{2}\left(\tau^{3} \delta_{g, 0}-\tau \delta_{g, 1}\right) .
$$

We claim that the functions $F_{g}$ are given by

$$
F_{g}(\tau)=-\operatorname{Li}_{3-2 g}\left(e^{\tau}\right)+ \begin{cases}-\frac{\tau^{2}}{2} \log (-\tau)-\frac{\tau^{3}}{12}+\frac{3 \tau^{2}}{4}-\frac{\pi^{2} \tau}{6}+\zeta(3) & \text { if } g=0, \\ -\frac{\tau}{2}-\log (-\tau) & \text { if } g=1, \\ (2 g-3) ! \tau^{2-2 g}-\frac{B_{2 g-2}}{2 g-2} & \text { if } g \geq 2 .\end{cases}
$$

Theorem 1.4 follows easily from equations (6.9) and (6.10).

It remains to prove (6.10). Equation (6.8) and (6.3) implies that for $g=1$ we have

$$
F_{1}(\tau)=f(\tau)=-\operatorname{Li}_{1}\left(e^{\tau}\right)-\frac{\tau}{2}-\log (-\tau) .
$$

From equation (6.8), it is easy to see that for $g \geq 1$ we have

$$
F_{g}(\tau)=\partial_{\tau}^{2 g-2} F_{1}(\tau)-(2 g-2) ! b_{2 g-2} .
$$

Since for all $\alpha \in \mathbb{R}$ we have

$$
\partial_{\tau} \operatorname{Li}_{\alpha}\left(e^{\tau}\right)=\operatorname{Li}_{\alpha-1}\left(e^{\tau}\right) .
$$

Equations (6.11) and (6.12) imply that for $g \geq 2$ we have:

$$
F_{g}(\tau)=-\operatorname{Li}_{3-2 g}\left(e^{\tau}\right)+(2 g-3) ! \tau^{2-2 g}-\frac{B_{2 g-2}}{2 g-2},
$$

where we have used the fact that $(2 g-2) ! b_{2 g}=B_{2 g} /(2 g-2)$.

When $g=0$, equation (6.8) implies that

$$
F_{0}(\tau)=\partial_{\tau}^{-2} f(\tau)
$$

up to a linear function of $\tau$, where $\partial_{\tau}^{-1}$ denotes integration with respect to $\tau$. Integrating (6.13) twice using (6.4), and matching the first three coefficients of the Taylor series of both sides at $\tau=0$ implies (6.10) for $g=0$. This concludes the proof of Theorem 1.4.

An alternative proof of equation (6.10) follows from Gopakumar-Vafa [31, Appendix]. See also $[45,46]$.

Yet another proof of equation (6.10) follows from [13, Proposition 1].

Remark 6.1. The reader may compare Theorem 1.4 with the BPS formulation of [31] and [46]. 


\section{$7 \quad$ Future directions}

\subsection{Analyticity of the free energy of a matrix model}

The notion of free energy exists for $U(N)$ gauge theories where $N$ is arbitrary. Toy models of those theories are the so-called matrix models studied by the French school $[12,10]$. In a future publication [26], we will study the analytic properties of the free energy of a matrix/multimatrix model with arbitrary potential. On the other hand, it was shown in [25] that given a closed 3-manifold $M$ presented by surgery on a framed link $L$ in $S^{3}$, there exists a multi-trace potential $V_{L}$ whose free energy coincides with the free energy of $M$. This observation gives geometric examples of different potentials with equal free energies. Combined with the future work of [26], this may give another proof of our main Theorem 1.1.

\subsection{Analytic continuation of the free energy in the complex plane}

This section is motivated by the questions that were raised in [23]. Fix a closed 3-manifold $M$, and let $\rho_{M, g}$ denote the radius of convergence of the power series $F_{\mathcal{M}, g}(\tau)$ at $\tau=0$. Theorem 1.1 implies that $\inf \left\{\rho_{M, g} \mid g \geq 0\right\}>0$.

Question 7.1. Fix a closed 3-manifold $M$. Is it true that the radius of convergence of $F_{M, g}(\tau)$ at $\tau=0$ is independent of $g$ ? Do the power series $F_{M, g}(\tau)$ admit analytic continuation as multivalued analytic functions in the complex plane minus a set of singularities, independent of $g$ ?

Notice that if we replace the closed 3-manifold $M$ by a knot $K$, and consider its free energy from the loop expansion of $K$, then the answer to this question is positive. This was discussed in Section 1.2. In that case, $F_{K, n}(\tau)$ are rational functions of $e^{\tau}$ and have analytic continuation in the complex plane minus the logarithms of the roots of the Alexander polynomial of $K$.

Question 7.2. Can you compute the free energy (or even its planar limit) for any closed hyperbolic manifold?

\subsection{The double scaling limit}

In the physics literature, it is customary to expect that $F_{g}(\tau)$ has an expansion around a $g$ independent singularity $\tau_{0}$ as follows:

$$
F_{g}(\tau)=\left(\tau-\tau_{0}\right)^{\gamma g+\delta} c_{g}(2 g) !+O\left(\left(\tau-\tau_{0}\right)^{\gamma g+1}\right) .
$$

In that case, one considers the single-variable power series

$$
f(x)=\sum_{g=0}^{\infty} c_{g}(2 g) ! x^{g},
$$

which is called the double scaling limit of $F$. In the case of matrix models, 2-dimensional gravity and random matrices, it turns out that $f(x)$ satisfies non-linear differential equations which are a specialization of the KP hierarchy. For a lengthy discussion, see [16], [55, Section 4] and references therein.

Question 7.3. Does the double scaling limit of the free energy of a closed 3-manifold satisfy a specialization of the KP hierarchy? 


\subsection{The $O(N)$ and $S p(N)$ theories}

In the present paper, we defined the free energy of a closed 3-manifold using the $U(N)$ gauge theory. We could have used the $O(N)$ or the $S p(N)$ gauge theory. In that case, the weight systems $W_{\mathfrak{o}_{N}}$ and $W_{\mathfrak{s p}_{N}}$ lead to trivalent graphs that embed to unoriented surfaces, see [2]. Theorem 1.1 holds in that case, assuming an asymptotic formula for the number of rooted unoriented maps; see Remark 4.1. A precise asymptotic expansion for the constants $p_{g}$ of Remark 4.1 will be discussed in a separate publication [27].

\section{A Asymptotics of $t_{g}$ and Painlevé I}

In this Appendix we show that the recursion relation for $t_{g}$ found in [7] is closely related to an asymptotic, formal solution to the Painlevé I differential equation. This makes possible to refine the asymptotic behavior obtained in [7].

We first recall some results from [7]. The asymptotics of $t_{g}$ is obtained from the asymptotics of two auxiliary sequences. The first one is $f_{g}$, which is defined by the recursion

$$
f_{g}=\frac{\sqrt{6}}{96}(5 g-4)(5 g-6) f_{g-1}+6 \sqrt{6} \sum_{h=1}^{g-1} f_{h} f_{g-h},
$$

and the initial condition

$$
f_{0}=-\frac{\sqrt{6}}{72}
$$

The sequence $t_{g}$ is related to $f_{g}$ by

$$
f_{g}=24^{-3 / 2} 6^{g / 2} \Gamma\left(\frac{5 g-1}{2}\right) t_{g} .
$$

Moreover, [7] introduces another sequence $u_{g}$, defined by

$$
u_{g}=f_{g}\left(\frac{25 \sqrt{6}}{96}\right)^{-g} \frac{6 \sqrt{6}}{[1 / 5]_{g}[4 / 5]_{g-1}} .
$$

Using (A.1), it is shown that $u_{g}$ approaches a constant $K$ as $g \rightarrow \infty$, and this leads to the main result of [7] concerning the asymptotics of $t_{g}$. The constant $K$ is in principle only known numerically.

To start our analysis, we define yet another sequence $a_{g}$ by the relation

$$
f_{g}=f_{0} C^{g} a_{g}, \quad C=\frac{\sqrt{6}}{2},
$$

so that the recursion (A.1) becomes

$$
a_{g}=\frac{25(g-1)^{2}-1}{48} a_{g-1}-\frac{1}{2} \sum_{\ell=1}^{g-1} a_{\ell} a_{g-\ell}, \quad a_{0}=1 .
$$

We now consider the formal power series

$$
\phi_{0}(z)=z^{\frac{1}{2}} \sum_{g=0}^{\infty} a_{g} z^{-5 g / 2} .
$$


It is then easy to see that the recursion (A.6) implies that $\phi_{0}(z)$ satisfies the following differential equation

$$
f^{2}-\frac{1}{6} f^{\prime \prime}=z,
$$

which is the well-known Painlevé I equation. Therefore, the differential equation presented at the end of [7] is Painlevé I in disguise.

We can now use properties of Painlevé I to give a precise description of the asymptotics of $a_{g}$, and in turn of $f_{g}$ and $t_{g}$. This follows from the general results of [15] together with the computations of [18] for Painlevé I; see also [38, Section 3]. We will then content ourselves with a statement of the asymptotic behavior of $a_{g}$ and a sketch of the main ideas.

The coefficient $a_{g}$ has the following asymptotic behavior as $g \rightarrow \infty$ :

$$
a_{g} \sim \frac{A^{-2 g+\frac{1}{2}}}{\pi} \Gamma\left(2 g-\frac{1}{2}\right) S\left\{1+\sum_{l=1}^{\infty} \frac{\mu_{l} A^{l}}{\prod_{k=1}^{l}(2 g-1 / 2-k)}\right\} .
$$

In this expression,

$$
A=\frac{8 \sqrt{3}}{5}, \quad S=-\frac{3^{\frac{1}{4}}}{2 \sqrt{\pi}}
$$

and the $\mu_{l}$ are defined by the recursion relation

$$
\mu_{l}=\frac{5}{16 \sqrt{3} l}\left\{\frac{192}{25} \sum_{k=0}^{l-1} \mu_{k} a_{(l-k+1) / 2}-\left(l-\frac{9}{10}\right)\left(l-\frac{1}{10}\right) \mu_{l-1}\right\}, \quad \mu_{0}=1 .
$$

It is understood here that $a_{n / 2}=0$ if $n$ is not an even integer, otherwise the coefficient $a_{n / 2}$ is given by the recursion (A.6). The quantities $S$ and $\mu_{l}$ have a very clear interpretation in terms of the differential equation (A.8). It is known (see for example [18]) that the Painlevé I equation exhibits a non-linear version of the Stokes phenomenon across the line $\operatorname{Arg} z=0 . S$ turns out to be the corresponding Stokes parameter. The exact value of $S$ was first obtained in [37] using the Riemann-Hilbert approach, further justified by [19]. A calculation of the Stokes parameter using a Borel summation approach and WKB was given by [53]. The coefficients $\mu_{l}$ arise as follows. The Painlevé I equation admits a so-called formal trans-series solution of the form

$$
\phi(z)=\phi_{0}(z)+z^{\frac{1}{2}} \sum_{\ell=1}^{\infty} \xi^{\ell} \phi_{\ell}(z), \quad \xi=C z^{-5 / 8} \mathrm{e}^{-A z^{5 / 4}},
$$

where $\phi_{0}(z)$ is the formal power series (A.7), $C$ is a constant and $\phi_{\ell}(z)$ are formal power series of the form

$$
\phi_{\ell}(z)=\sum_{k=0}^{\infty} \phi_{\ell, k} z^{-5 k / 4}
$$

normalized by $\phi_{1}(0)=1$. The Painlevé I equation gives a series of recursion relations for the coefficients $\phi_{\ell, k}$ in terms of $a_{n}$ and $\phi_{\ell^{\prime}, k^{\prime}}$ with $\ell^{\prime}<\ell$. The coefficients of the first series in the trans-series, $\phi_{1, k}$, satisfy precisely the recursion (A.11), therefore $\mu_{l}=\phi_{1, l}$. One finds, for the very first terms,

$$
\phi_{1}(z)=1-\frac{5}{64 \sqrt{3}} z^{-\frac{5}{4}}+\frac{75}{8192} z^{-\frac{5}{2}}-\frac{341329}{23592960 \sqrt{3}} z^{-\frac{15}{4}}+\cdots .
$$


The fact that the value of the Stokes parameter $S$ together with the coefficients of the first trans-series correction $\phi_{1}(z)$ lead to an asymptotic behavior of the form (A.9) has been known for a long time in the physics literature on large-order behavior in perturbation theory; see for example [44]. A rigorous proof for the case of Painlevé I was given in [38, Section 3]. For a general approach to this problem for a class of generic non-linear ODEs, see [15]. Using these results we can now revisit the asymptotics of the sequences $u_{g}$ and $t_{g}$. One finds indeed that

$$
u_{g} \rightarrow K
$$

as $g \rightarrow \infty$. The constant $K$ can now be determined exactly from the asymptotics of $a_{g}$. Its value is,

$$
K=\sqrt{\frac{3}{5}} \frac{\Gamma(1 / 5) \Gamma(4 / 5)}{4 \pi^{2}}=0.10486898772254091800 \ldots
$$

In fact, the numerical approximation to the value of $K$ presented in [7] is not very precise, and can be substantially improved by using standard techniques like Richardson transforms. Of course, the results above give all the subleading corrections to the asymptotics of $u_{g}$ (and $t_{g}$ ) in powers of $1 / \mathrm{g}$.

Let us use the Painlevé equation to answer a question posed by [7] concerning the sequence $t_{g}$. The question asks whether the sequence $\left(t_{g}\right)$ is holonomic, i.e., satisfies a linear recursion in $g$ with coefficients polynomials in $g$. It is easy to show that if $t_{g}$ is holonomic, then the generating series $\phi_{0}(z)$ satisfies a linear ODE with coefficients polynomials in $z$. It follows that $\phi_{0}(z)$ (or more precisely, its Borel transform in a suitable ray) has analytic continuation as a multivalued analytic function in $\mathbb{C}$ minus a finite set of singularities. On the other hand, it is known that any (truncated) solution of Painlevé $I$ is meromorphic (see for example [11, 36]), with infinitely many singularities that are asymptotic to one of the rays $\arg (z)=2 \pi k / 5$ for $k=1, \ldots, 5$ (see for example, [11, 19]).

Corollary A.1. The sequence $\left(t_{g}\right)$ is not holonomic.

We end this Appendix by pointing out some connections between the results of $[7,32]$ and the physics literature on matrix models and two-dimensional gravity. It has been known since the pioneering work of $[12,10]$ that matrix models are a useful tool to count maps of genus $g$ and with $n$ vertices. The resulting generating functions at fixed genus $g$ are all analytic functions in the vertex-counting parameter, and they have a finite radius of convergence which is common to all of them. The sequence $f_{g}$ in [7] is given indeed by the coefficients of the leading singularities for one of these generating functions. It is known however from the physics literature on the so-called "double scaling limit" that these coefficients are governed by the Painlevé I equation (see [16]), and in fact, as we have seen, this is the differential equation governing the sequence $f_{g}$. A study of the asymptotics of various generating functions appearing in matrix models, including the Painlevé I equation, can be found in [47].

\section{Acknowledgements}

Much of the paper was conceived during conversations of the first and third authors in Geneva in the spring of 2008. S.G. wishes to thank M.M. and R. Kashaev for the wonderful hospitality, E. Witten who suggested that we look at the $U(N)$ Chern-Simons theory for arbitrary $N$ and A. Its for enlightening conversations on the Riemann-Hilbert problem. 


\section{References}

[1] Aoki T., Kawai T., Koike T., Takei Y., On the exact WKB analysis of operators admitting infinitely many phases, Adv. Math. 181 (2004), 165-189.

[2] Bar-Natan D., On the Vassiliev knot invariants, Topology 34 (1995), 423-472.

[3] Bar-Natan D., Garoufalidis S., On the Melvin-Morton-Rozansky conjecture, Invent. Math. 125 (1996), 103-133.

[4] Bar-Natan D., Lawrence R., A rational surgery formula for the LMO invariant, Israel J. Math. 140 (2004), 29-60, math.GT/0007045.

[5] Bar-Natan D., Garoufalidis S., Rozansky L., Thurston D., The Aarhus integral of rational homology 3spheres. I. A highly non trivial flat connection on $S^{3}$, Selecta Math. (N.S.) 8 (2002), 315-339, q-alg/9706004. Bar-Natan D., Garoufalidis S., Rozansky L., Thurston D., The Aarhus integral of rational homology 3spheres. II. Invariance and universality, Selecta Math. (N.S.) 8 (2002), 341-371, math.QA/9801049.

Bar-Natan D., Garoufalidis S., Rozansky L., Thurston D., The Aarhus integral of rational homology 3spheres. III. Relation with the Le-Murakami-Ohtsuki invariant, Selecta Math. (N.S.) 10 (2004), 305-324, math.QA/9808013.

[6] Bender E.A., Canfield E.R., The asymptotic number of rooted maps on a surface, J. Combin. Theory Ser. A 43 (1986), 244-257.

[7] Bender E.A., Gao Z., Richmond L.B., The map asymptotics constant $t_{g}$, Electron. J. Combin. 15 (2008), no. 1, paper 51,8 pages.

[8] Benna M.K., Benvenuti S., Klebanov I.R., Scardicchio A., A test of the AdS/CFT correspondence using high-spin operators, hep-th/0611135.

[9] Beisert N., Eden B., Staudacher M., Transcendentality and crossing, J. Stat. Mech. 2007 (2007), P01021, 30 pages, hep-th/0610251.

[10] Bessis D., Itzykson C., Zuber J.B., Quantum field theory techniques in graphical enumeration, Adv. in Appl. Math. 1 (1980), 109-157.

[11] Boutroux P., Recherches sur les transcendantes de M. Painlevé et l'étude asymptotique des équations différentielles du second ordre, Ann. Sci. École Norm. Sup. (3) 30 (1913), 255-375.

[12] Brézin E., Itzykson C., Parisi G., Zuber J.B., Planar diagrams, Comm. Math. Phys. 59 (1978), 35-51.

[13] Cohen H., Lewin L., Zagier D., A sixteenth-order polylogarithm ladder, Experiment. Math. 1 (1992), 25-34.

[14] Costin O., Garoufalidis S., Resurgence of the fractional polylogarithms, Math. Res. Lett., to appear, math.CA/0701743.

[15] Costin O., Kruskal M., Optimal uniform estimates and rigorous asymptotics beyond all orders for a class of ordinary differential equations, Proc. Roy. Soc. London Ser. A 452 (1996), no. 1948, 1057-1085, math.CA/0608412.

[16] Di Francesco P., Ginsparg P., Zinn-Justin J., 2D gravity and random matrices, Phys. Rep. 254 (1995), no. 1-2, 133 pages, hep-th/9306153.

[17] Eynard B., Zinn-Justin J., Large order behaviour of 2D gravity coupled to $D<1$ matter, Phys. Lett. B 302 (1993), 396-402, hep-th/9301004.

[18] Fokas A.S., Its A.R., Kitaev A.V., The isomonodromy approach to matrix models in 2D quantum gravity, Comm. Math. Phys. 147 (1992), 395-430.

[19] Fokas A.S., Its A.R., Kapaev A., Novokshenov V.Yu., Painlevé transcendents. The Riemann-Hilbert approach, Mathematical Surveys and Monographs, Vol. 128, American Mathematical Society, Providence, RI, 2006.

[20] Garoufalidis S., Lê T.T.Q., Gevrey series in quantum topology, J. Reine Angew. Math. 618 (2008), 169-195, math.GT/0609618.

[21] Garoufalidis S., Lê T.T.Q., Asymptotics of the colored Jones function of a knot, math.GT/0508100.

[22] Garoufalidis S., Difference and differential equations for the colored Jones function, J. Knot Theory Ramifications 17 (2008), 495-510, math.GT/0306229.

[23] Garoufalidis S., Chern-Simons theory, analytic continuation and arithmetic, Acta Math. Vietnam., to appear, arXiv:0711.1716.

[24] Garoufalidis S., Habegger N., The Alexander polynomial and finite type 3-manifold invariants, Math. Ann. 316 (2000), 485-497, q-alg/9708002. 
[25] Garoufalidis S., Mariño M., On Chern-Simons matrix models, math.GT/0601390.

[26] Garoufalidis S., Mariño M., in preparation.

[27] Garoufalidis S., Mariño M., in preparation.

[28] Garoufalidis S., Rozansky L., The loop of the Kontsevich integral, the null-move and S-equivalence, Topology 43 (2004), 1183-1210, math.GT/0003187.

[29] Gao Z.C., A pattern for the asymptotic number of rooted maps on surfaces, J. Combin. Theory Ser. A 64 (1993), 246-264.

[30] Gao Z.C., The number of rooted triangular maps on a surface, J. Combin. Theory Ser. B 52 (1991), $236-249$.

[31] Gopakumar R., Vafa C., M-theory and topological strings-I, hep-th/9809187.

[32] Goulden I.P., Jackson D.M., The KP hierarchy, branched covers, and triangulations, Adv. Math., to appear, arXiv:0803.3980.

[33] Habegger N., Masbaum G., The Kontsevich integral and Milnor's invariants, Topology 39 (2000), 1253-1289.

[34] Habegger N., Thompson G., The universal perturbative quantum 3-manifold invariant, Rozansky-Witten invariants and the generalized Casson invariants, Acta Math. Vietnam., to appear, math.GT/9911049.

[35] 't Hooft G., On the convergence of planar diagram expansions, Comm. Math. Phys. 86 (1982), 449-464.

[36] Joshi N., Kruskal M.D., A direct proof that solutions of the six Painlevé equations have no movable singularities except poles, Stud. Appl. Math. 93 (1994), 187-207.

[37] Kapaev A.A., Asymptotic behavior of the solutions of the Painlevé equation of the first kind, Differentsial'nye Uravneniya 24 (1988), 1684-1695 (English transl.: Differential Equations 24 (1988), 1107-1115).

[38] Kapaev A.A., Quasi-linear stokes phenomenon for the Painlevé first equation, J. Phys. A: Math. Gen. 37 (2004), 11149-11167, nlin.SI/0404026.

[39] Kupergberg G., Thurston D.P., Perturbative 3-manifold invariants by cut-and-paste topology, math.GT/9912167.

[40] Kuriya T., On the LMO conjecture, arXiv:0803.1732.

[41] Lê T.T.Q., Murakami J., Ohtsuki T., On a universal perturbative invariant of 3-manifolds, Topology 37 (1998), 539-574.

[42] Lê T.T.Q., On perturbative PSU(n) invariants of rational homology 3-spheres, Topology 39 (2000), 813-849, math.GT/9802032.

[43] Lê T.T.Q., Quantum invariants of 3-manifolds: integrality, splitting, and perturbative expansion, Topology Appl. 127 (2003), 125-152, math.QA/0004099.

[44] Le Guillou J.C., Zinn-Justin J. (Editors), Large order behavior of perturbation theory, North-Holland, Amsterdam, 1990.

[45] Mariño M., Chern-Simons theory, matrix integrals and perturbative three-manifold invariants, Comm. Math. Phys. 253 (2005), 25-49, hep-th/0207096.

[46] Mariño M., Chern-Simons theory and topological strings, Rev. Modern Phys. 77 (2005), 675-720, hep-th/0406005.

[47] Mariño M., Schiappa R., Weiss M., Nonperturbative effects and the large-order behavior of matrix models and topological strings, Commun. Number Th. Phys. 2 (2008), 349-419, arXiv:0711.1954.

[48] Oesterlé J., Polylogarithmes, Séminaire Bourbaki, Vol. 1992/93, Astérisque no. 216 (1993), Exp. no. 762, 49-67.

[49] Ohtsuki T., A polynomial invariant of rational homology 3-spheres, Invent. Math. 123 (1996), $241-257$.

[50] Ohtsuki T., Quantum invariants. A study of knots, 3-manifolds, and their sets, Series on Knots and Everything, Vol. 29, World Scientific Publishing Co., Inc., River Edge, NJ, 2002.

[51] Rozansky L., The universal $R$-matrix, Burau representation and the Melvin-Morton expansion of the colored Jones polynomial, Adv. Math. 134 (1998), 1-31.

[52] Takata T., On quantum PSU(n)-invariants for lens spaces, J. Knot Theory Ramifications 5 (1996), 885-901.

[53] Takei Y., On the connection formula for the first Painlevé equation - from the viewpoint of the exact WKB analysis, Surikaisekikenkyusho Kokyuroku 931 (1995), 70-99.

[54] Turaev V., The Yang-Baxter equation and invariants of links, Invent. Math. 92 (1988), 527-553.

[55] Witten E., Two-dimensional gravity and intersection theory on moduli space, in Surveys in Differential Geometry (Cambridge, MA, 1990), Lehigh Univ., Bethlehem, PA, 1991, 243-310. 